\title{
Féeries
}

Études sur le conte merveilleux, XVII $-\mathrm{XIX}{ }^{\mathrm{e}}$ siècle

16 | 2020

Le conte, les mythes antiques, la Bible

\section{Les contes, les mythes, la Bible, merveilleuses littératures}

Pierre-Emmanuel Moog

\section{OpenEdition}

1 Journals

Édition électronique

URL : https://journals.openedition.org/feeries/2497

DOI : $10.4000 /$ feeries. 2497

ISSN : 1957-7753

Éditeur

UGA Éditions/Université Grenoble Alpes

Édition imprimée

ISBN : 978-2-37747-194-2

ISSN : $1766-2842$

Référence électronique

Pierre-Emmanuel Moog, "Les contes, les mythes, la Bible, merveilleuses littératures », Féeries [En ligne], 16 | 2020, mis en ligne le 26 novembre 2020, consulté le 10 décembre 2021. URL : http:// journals.openedition.org/feeries/2497 ; DOI : https://doi.org/10.4000/feeries.2497

Ce document a été généré automatiquement le 10 décembre 2021.

(c) Féeries 


\title{
Les contes, les mythes, la Bible, merveilleuses littératures
}

\author{
Pierre-Emmanuel Moog
}

Disons-le d'emblée, ce dix-septième numéro de Féeries sur « les contes, les mythes, la Bible " est, à certains égards, un échec. Il se donnait pour ambition de faire se rencontrer les spécialistes des contes avec les spécialistes des littératures antiques (hellénistes, biblistes, ou - pourquoi non - spécialistes des études coraniques) mais ces derniers n'ont pas répondu à l'appel. Les raisons tiennent certainement aux cloisons trop étanches qui ont été érigées entre les corpus, alors que nos outils d'analyse et nos matières textuelles mêmes auraient beaucoup à échanger. Cependant, bien heureusement, les conteux, eux, se sont emparés de la perspective, et se sont efforcés de croiser les genres: textes sacrés, mythes, légendes, contes littéraires et contes de tradition orale, et même incantations liturgiques. Ce numéro est-il donc aussi une réussite? Nous vous laissons l'apprécier.

2 Mais, en premier lieu, pourquoi escompter une rencontre fructueuse entre des littératures antiques (mythologie, Bible) et des littératures contiques (classiques ou populaires), entre leurs spécialistes, leurs textes et leurs herméneutiques? Car nous parlons bien de littérature, c'est-à-dire de textes se retrouvant, d'une manière ou d'une autre, écrits. Certains ont affirmé que les contes sont le résultat de la dégradation des mythes. Peut-être. Cependant notre problématique, ici, n'est pas généalogique, mais narrative.

\section{Mirabilia}

En un mot, les littératures antiques et contiques qui nous occupent possèdent le merveilleux en partage. On y trouve des métamorphoses, d'une forme humaine à une forme animale et vice-versa, de la vie à la mort à la vie (quelques endormissements comateux, comme la Belle au fond des bois, et quelques résurrections stupéfiantes avec Elie, Elisée ou Jésus, qui se multiplieront dans les Kinder- und Hausmärchen), des objets transformateurs (comme la baguette de Moïse ou de Circé, et le bâton des fées, ou 
l'inverse, n'est-ce pas ?). On y passe d'un monde à l'autre, catabase \& anabase. Les héros y sont assistés par des adjuvants (un ou des dieux, des anges et des fées, marraines ou pas) et se confrontent à des antagonistes (un ou des dieux, géants, cyclopes, sorciers, ogres et autres diables...).

Une fois de plus, après tant d'autres, qu'est-ce que le merveilleux? La fameuse distinction d'avec le fantastique, de Roger Caillois à Tzvetan Todorov, reste opérante, même si sans doute moins tranchée que les concepts théoriques le laissent accroire. Car il est des nombreux contes ou des textes antiques où l'apparition du surnaturel ne va pas forcément de soi, et peut même un tantinet étonner. Pour la Bible hébraïque, les théophanies sont somme toute peu nombreuses, et diversement ressenties par les personnages: Abram accepte comme une évidence la parole de Dieu (Gn 12), mais Moïse est stupéfait par des signes extraordinaires (Ex 3), Gédéon est carrément sceptique (Jg 6) et Manoah perplexe (Jg 13). Dans les contes de Perrault, l'épouse de la Barbe bleue résiste à accepter le caractère féerique de la clé ensanglantée, en dépit de taches réapparaissantes.

D'autre part, il faudrait sans doute distinguer un merveilleux intra-diégétique d'un merveilleux extra-diégétique. Dans le premier, les personnages perçoivent sans s'étonner, plus ou moins donc, les phénomènes surnaturels, et dans le second, le narrateur n'indique pas clairement au lecteur le statut imaginaire (hallucinatoire, récit de rêve) ou « réel » du surnaturel qui semble être intervenu dans le récit. Dans la Bible hébraïque, le combat de Jacob (Gn 32) ou celui de Moïse (Ex 4) relèvent de ce second merveilleux, quasi fantastique, tout comme, dans le « Riquet à la houppe » de Perrault, l'apparition chtonienne et la transformation du difforme en bel homme.

6 En outre, le surnaturel est moins généralisé qu'on ne le dit, aussi bien dans les littératures antiques que contiques, car il ne s'agit pas d'invraisemblance généralisée, que l'on trouverait éventuellement dans le fantastique, certaines formes de fantasy, ou plus sûrement le surréalisme (pour atteindre poétiquement d'autres niveaux de conscience, sans doute). En fait, précisément en opposition avec le fantastique, si l'occurrence surnaturelle est structurante dans les genres merveilleux, elle n'est pas ce autour de quoi tournent les récits. Au contraire, les phénomènes surnaturels sont plutôt des instruments narratifs pour accélérer et aller à l'essentiel: (se) métamorphoser revient à faire ressortir littéralement le tréfonds de l'âme d'une personne à la surface; se transporter loin en quelques enjambées bottées revient à s'adjoindre des moyens extraordinaires en évitant d'en discuter les détails techniques dilatoires. Du merveilleux conçu comme un vaste terrain d'expérimentation permettant les multiples combinaisons d'expériences de pensées narratives...

D'ailleurs, Catherine Bernard préconisait en 1696 «des règles pour ces sortes d'histoires dont voici les deux principales : que les aventures fussent toujours contre la vraisemblance, et les sentiments toujours naturels. On jugea que l'agrément de ces

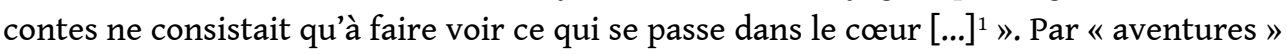
il faut entendre les événements de l'histoire et par "sentiment» ce que nous appellerions la psychologie des personnages. Sur le premier point, il faut reconnaître que les intrigues des conteuses de la décennie 1690, sous-genre en tant que tel, sont particulièrement échevelées et rebondissantes (d'où leurs longueurs au propre comme au figuré), mais peu représentatives sur ce point des contes merveilleux dans leur ensemble. Quant aux «sentiments", l'auteur professe avec justesse que les récits merveilleux sont, quoiqu'on dise, traversés de part en part par la condition 
psychologique humaine, non seulement dans les actions des personnages (comme le veulent les théories d'André Jolles ou Max Lüthi) mais aussi par des discrets mais significatifs détails disséminés çà et là, notamment dans les micro-différences des répétitions si caractéristiques de la poétique des contes. Finalement, si l'on prête attention aux moindres sous-entendus, selon leurs contextes, les personnages font l'objet d'une caractérisation psychologique approfondie.

Il existe un paradoxe du merveilleux contique, spécifiquement. Car ce procédé, non pas accessoire, mais instrumental, créé des images si fortes, fulgurantes même, que par un tour proprement démoniaque, le merveilleux finit par fasciner et sidérer le lecteur, détournant son attention, au passage, et souvent malgré lui, des éléments plus essentiels du récit. Il reste à déterminer dans quelle mesure ce paradoxe joue également dans les littératures antiques pour lesquelles le surnaturel possède, aussi, une fonction mythique ou théologique.

Derechef, qu'est-ce donc que le merveilleux dans les récits merveilleux ? Il ne se limite pas à l'intervention du surnaturel, finalement limité. L'opinion courante est que les contes et la Bible hébraïque, et dans une moindre mesure les textes gréco-latins, sont invraisemblables. Comme l'exprime joliment Anatole France, «ces contes sont absurdes. S'ils n'étaient pas absurdes, ils ne seraient pas charmants ${ }^{2}$ ». Pourtant, si l'on évite de trop regarder le surnaturel en face, afin de ne pas en rester pétrifié, ces littératures sont plus vaguement irréalistes qu'absurdes ou invraisemblables. Elles mettent en œuvre une formule commune du merveilleux dont nous pouvons préciser les ingrédients :

- un contexte spatio-temporel sous-déterminé (mais bien sous-jacent);

- des conditions sociales extrêmes (beaucoup de rois \& reines, de princes \& princesses, des bergères et des bûcherons, mais aussi des voyous, voleurs, chenapans, et même quelques quidam) ;

- des personnages au caractère hyperbolique (mais pas forcément manichéen - ils sont trop blanc et trop noir, et pas forcément tout blanc ou tout noir, dotés d'une volonté et d'une témérité exceptionnelles, bigger than life comme en raffole le cinéma américain à la limite du réalisme);

- des situations sociales et familiales exacerbées, au-delà même de l'extravagance romanesque ;

- une bonne dose de surnaturel, certes ;

- des coïncidences extraordinaires très improbables (mais pas strictement impossibles);

- et enfin, une orientation positive, surtout pour les contes (nous reviendrons sur ce point).

\section{Narratio}

10 Au-delà de ces mirabilia, les littératures antiques et contiques partagent de nombreux autres aspects structurels. Elles s'échangent des thèmes, des motifs, des traits narratifs. Elles se les prêtent et se les empruntent, avec intérêts³.

$11 \mathrm{Au}$ commencement, il y a un éloignement. La fonction proppienne initiale d'éloignement n'est pas réservée à la fillette à la coiffe rouge chargée de se rendre au prochain village à travers bois, ni aux contes en général. Adam et Ève, déjà, étaient chassés du cocon paradisiaque, Abram a reçu un appel à quitter la maison de son père, les Achéens se mobilisent pour leur grande expédition trans-égéenne... La recette est la même : isoler les protagonistes de leur environnement d'origine, de leurs adjuvants 
(maman aimant follement, Dieu omniprésent en Éden) ou antagonistes habituels, les lancer dans un monde vaste et résistant.

Des rivalités fraternelles féroces sont mises en scène. Souvent le faible/dominé/cadet affronte le fort/dominant/aîné et s'en sort par la ruse ${ }^{4}$. Celle-ci est également l'objet d'un paradoxe narrativement prolifique. Souvent, la ruse est une astuce ou une tromperie si simple que le lecteur l'aurait trouvée lui-même, se dit-il après coup... mais qui surprend toujours, semblant n'obéir à aucune loi. Elle permet donc une créativité narrative sans cesse renouvelée. Un cas particulier de la ruse et de l'astuce est l'énigme, récurrente dans les contes et dans la mythologie, plus rare dans la Bible hébraïque (on peut penser néanmoins à l'énigme de Samson ou au jugement de Salomon).

transgressions d'interdits anthropologiques fondamentaux sont fréquents dans les littératures antiques comme contiques: inceste, cannibalisme, parricide, infanticide (du non-sacrifice d'Isaac ou de la fille de Jephté à celui d'Iphigénie, jusqu'au motif de l'enfant exposés). On retrouve aussi communément des interdits plus spécifiques, a priori arbitraires, énoncés par un protagoniste détenant l'autorité : ne pas manger (certains fruits en Gn 2), ne pas se retourner (la famille de Loth en Gn 19 ou Orphée), ne pas ouvrir (de Pandore à Psyché à la Barbe bleue ${ }^{6}$ )... On comprend que le merveilleux soit le cadre naturel pour ces expérimentations narratives, par la possibilité à la fois de représenter des situations-limites, et de sanctionner automatiquement (pour ne pas dire magiquement) la désobéissance qui ne peut rester impunie (le crime presque parfait du whodunit ou le délit plus que parfait d'un Arsène sont aux antipodes narratives).

Sur le plan formel, les littératures antiques et contiques partagent la forme courte. En effet, comme on l'a vu, le merveilleux permet une littérature condensée, qui n'a pas à se perdre dans les détails, les péripéties et les circonstances romanesques pour générer des situations expérimentales. Même si, pour les littératures antiques, certaines compilations ont réussi à agréger, parfois avec bonheur (par hasard, la Bible hébraïque ou l'Odyssée), une multitude de courts récits, de motifs et de thèmes, qui conservent une relative autonomie. Mais la brièveté des récits n'est pas celle des histoires: contrairement aux fables qui s'arrêtent sur un événement ponctuel exemplaire, nos récits accompagnent les personnages sur un long parcours de vie, de sorte que le héros n'est pas toujours celui qui se définirait par sa moralité mais par sa durée de vie la plus longue ${ }^{7}$, et son éventuelle capacité à évoluer.

Autre point commun, lié au précédent : leur temporalité narrative. Les intrigues sont souvent représentées dans un ordre linéaire, mais surtout elles aménagent des rythmes narratifs souvent surprenants, avec des expositions accélérées par l'emploi de prototypes sociaux et la mise en relief du détail significatif, des ellipses vertigineuses et parfois ambiguës, et des pauses sur une scène avec de brefs dialogues et de rares descriptions ${ }^{8}$. Au-delà de la triplication des épreuves, l'usage des répétitions différentielles, qui n'est pas que la trace d'une oralité mais un puissant procédé de création de sens, traverse les littératures bibliques et contiques.

16 Autre point commun, justement : ces littératures proviennent sans doute en amont de traditions orales, au moins en partie, mais dans une mesure difficile à établir. Et, en aval, elles se donnent à lire à travers de nombreuses variantes. Quoique le texte biblique ait été fixé en canons, il subsiste quelques micro-variantes entre le texte massorétique, le texte samaritain, la Septante, la Vulgate, etc. Mais au-delà, les récits sont paraphrasés dans les Antiquités Juives de Flavius Josèphe, l'Exagôge d'Ezechiel le 
Tragique, les midrashim du Bereshit Rabba jusqu'au Coran lui-même, offrant un vaste terrain de jeux herméneutiques. Pareillement pour les récits mythologiques, dont les histoires peuvent se retrouver plus ou moins détaillées chez Eschyle, Euripide, Apollodore, Ovide, Stace, etc. Il en est bien sûr de même de nos contes, dont la critique se fait un devoir et un plaisir jouissif de suivre les généalogies littéraires ${ }^{9}$ ou les variantes d'un conte-type ${ }^{10}$.

D'ailleurs, les constructions différemment rythmées de ces récits merveilleux, quand ils tournent autour d'une même problématique, offrent parfois l'opportunité d'interpréter le silence d'un épisode d'un récit donné par le détail révélateur qu'en apporte un autre. Les récits merveilleux, au-delà des genres, forment un vaste réseau, qui peuvent dans une certaine limite se suppléer les uns aux autres, sans pour autant perdre l'autonomie de leur message global, théologique ou initiatique ${ }^{11}$.

Résultant de cette série de points communs entre littératures merveilleuses antiques et contiques, celles-ci sont parallèlement confrontées au paradoxe du succès : méprises et sottises. Les récits de la Bible hébraïque, les contes de Perrault et ceux des Grimm, en particulier, ont connu une fortune extraordinaire. Leur style apparemment simple et naïf y est pour beaucoup. Mais ce n'est qu'apparence, comme le montre le renouvellement des commentaires, de générations en générations. La densité des textes, leur poétique subtile a suscité des modes interprétatives se succédant les unes aux autres, sans plus aucune considération pour les textes réels. Le folklorisme, la psychanalyse, la critique marxiste ou féministe, par leur systématisme, ont fait preuve d'une ingéniosité souvent malheureuse face aux contes de Perrault. Déjà auparavant, l'Église, se réservant la critique biblique, avait fini par concevoir parmi les plus terribles des contre-sens imaginables, par son impact civilisationnel, celui du péché originel $^{12}$. Toujours l'Église (mais la part rabbinique n'est cependant pas à exclure dans ce long processus hagiographique), dans une expansion de la figure du saint des Évangiles aux patriarches de la Bible hébraïque, a élaboré un récit exemplaire en décalage complet avec le texte qui met en scène, en réalité, des personnages trop humains. Ces aberrations herméneutiques provoquent la rébellion d'esprits forts, comme Voltaire, qui se fourvoie néanmoins de cible, s'insurgeant avec mauvaise foi contre un texte à partir de la lecture catéchistique qu'il a dû subir. Un exemple, si l'on ose dire édifiant, est sa critique assassine mais malhonnête et surtout malavisée du cycle de Jacob ${ }^{13}$.

Les littératures contiques sont aussi dans une relation cette fois de filiation par rapport aux littératures antiques, concernant leur personnel merveilleux. L'ange et le diable issus de la Bible hébraïque, pour le premier peu fréquent et simple envoyé ponctuel de Dieu à l'apparence humaine, pour le second rarissime satan tentateur, sont transfigurés par les contes en prototypes récurrents, polyvalents et dotés des riches imaginaires visuels et colorés $^{14}$. De même, les cyclopes, géants, titans et autres monstres mythologiques se retrouvent en partie dans le personnage de l'ogre cannibale, mais diversement humanisés selon les auteurs contiques. L'anathème évangélique porté sur une princesse judéenne aux mœurs décadentes, et vengeresse, Hérodias, la transforme dans les croyances populaires pan-européennes en une figure diabolique ${ }^{15}$.

Des différences cependant marquent les littératures antiques et contiques. Les premières, par droit d'aînesse, sont macro-étiologiques (cosmogénèse, anthropogenèse, ethnogenèse), tandis que les secondes sont micro-étiologiques (parfois de manière anecdotique dans les légendes ou artificielle dans les contes). Les premières conservent, au-delà du sens narratif, un sens théologique ou philosophique superposé, tandis que 
les secondes sont plus initiatiques. Du fait de leur construction en cycles, les premières mettent en scène des ennemis jurés (Philistins, Amalécites), dont on ne vient jamais à bout et qui réapparaissent donc sans cesse, quand les secondes clôturent le récit par un châtiment cruel et définitif du méchant. Corrélativement, les premières peuvent difficilement garantir une issue perpétuellement heureuse à leurs héros, quand celle-ci n'est pas simplement dramatique du fait de cycles de vengeances, alors que les secondes dénouent une situation en quelques pages et assurent un destin stabilisé, par le mariage et l'ascension ou la restauration sociale, aux héros.

En guise de conclusion, forcément provisoire, à cette introduction, il ne peut être question de réduire les contes merveilleux à la Bible ou à la mythologie, et vice-versa. Des schèmes narratifs similaires les traversent, reflétant des enjeux anthropologiques communs à travers les millénaires et les aires culturelles, même si les sens des récits peuvent différer, en fonction de leur visée. Pourtant, l'étude de ces différences aussi est riche d'effets de sens pour les uns comme pour les autres. La difficulté est patente néanmoins : la nécessité de maîtriser des corpus si vastes et des langues si distinctes. La pluridisciplinarité est donc souhaitable. Il serait possible, par exemple, d'examiner et de comparer leurs personnels, leurs bestiaires, leurs objets magiques, leurs lieux-types, etc, pour en repérer de possibles invariants. Comme les auteurs de ce numéro de Féeries se sont autorisés à le faire, il s'agit de pratiquer des analyses croisées. Depuis l'ouvrage pionnier Folklore in Old Testament (1918) de James Frazer, ces tentatives sont encore rares, et nous ne pouvons que les encourager.

\section{NOTES}

1. C. Bernard, Cuvres, éd. F. Piva, Fasano/Paris, Schena/Nizet, 1903, p. 347-348.

2. A. France, Le Livre de mon ami, Paris, Calmann-Lévy, 1890, p. 270.

3. Comme le montre, dans ce numéro, les analyses reconfiguratives de Ute Heidmann («Vénus, la Barbe bleue et la Vierge Marie. Enquête intertextuelle et interculturelle sur une étrange filiation »), et l'étude des permanences des motifs de Gaëlle Le Guern («D'Éros à la Bête: la chambre des secrets ").

4. Comme on le verra, avec Patricia Eichel-Lojkine, dans ce numéro («Confondre les sages : échos évangéliques dans un conte facétieux de Straparola »).

5. Comme le montre l'étude de Bochra et Thierry Charnay dans ce numéro ( Le motif de « l'enfant exposé » : un cas type de la permanence des schémas mythiques par-delà les genres »).

6. Thème central de l'article de Ute Heidmann dans ce numéro.

7. Selon la formule heureuse de Nicole Belmont.

8. Comme le souligne Marc Hersant dans ce numéro (« Le conte voltairien : un parasite des textes sacrés »).

9. Voir l'article de Ute Heidmann dans ce numéro.

10. Voir l'article de Bochra et Thierry Charnay dans ce numéro.

11. Comme je tente de le montrer, dans ce numéro, avec des figures d'Invincibles révélant, plus ou moins, leur secret (« La faille de L'Invincible, de Samson à Kachtcheï »). 
12. La transgression de Gn 3 pourrait s'interpréter comme salutaire, à lire attentivement le texte, mais nous ne pouvons pas développer ici.

13. Comme le présente Marc Hersant dans ce numéro.

14. Comme le montre Christiane Connan-Pintado dans ce numéro ( L'ange et le diable, deux figures merveilleuses du personnel biblique $»)$.

15. On suivra cette évolution avec un étonnant éclectisme des sources dans l'article de Stamatios Zochios dans ce numéro ( La souveraine du lac gelé: variantes et altérations du mythe d'Hérodiade dans les croyances populaires européennes »). 